\title{
A pain perception comparison of intraoral dental anesthesia with 26 and 30 gauge needles in 6-12-year-old children
}

\author{
Alexander Asokan*, Arun Prasad Rao², G. Mohan², N. Venugopal Reddy², Krishna Kumar ${ }^{2}$ \\ ${ }^{1}$ Rishiraj Dental College and Research Centre, Bhopal, Madhya Pradesh, ${ }^{2}$ Rajah Muthiah Dental College and Hospital, \\ Department of Pedodonties and Preventive Dentistry, Chidambaram, Tamil Nadu, India
}

\begin{abstract}
The aim was to compare, in vivo the pain perception of children to local anesthesia (LA) using 26 gauge and 30 gauge needles with different age, sex, and visits. A total sample of 30 children (16 boys and 14 girls) who were undergoing routine dental procedures was selected for the study. Children were assigned into two groups: Group A: 12 children aged 6-8 years Group B: 18 children aged 9-12 years. Informed consents were obtained from parents. The objective and subjective behavioral parameters were evaluated by student $t$-test and Chi-squared analyses, and significance was idealized at $P<0.05$. The mean pain scores for 26 gauge needles was found to be 2.80 whereas for pain scores for 30 gauge needles were found to be 2.37 . The mean difference was found to be statistically significant $(P=0.001)$. The mean pain score in first visit for patients who received LA with 30 gauge needles was found to be 2.00, whereas for second visit was found to be 2.61. The mean pain difference was found to be statistically significant $(P=0.01)$. No significance was seen in relation to age and gender. The pain due to injection penetration might be controlled by using thinner gauge needles in spite of difference in pain perceptions. During a child's first dental visit, use of less pain provoking armamentarium would aid in relieving anxiety and desensitize the patient slowly.
\end{abstract}

Key words: Local Anaesthesia, Needle Gauge, Pain Perception

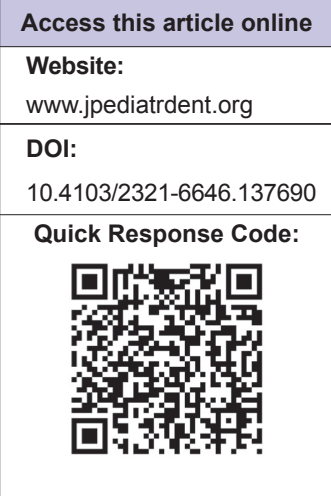

\section{INTRODUCTION}

$\mathrm{F}$ ear-related behaviors had long been recognized as the most difficult aspect of patient management and could be a barrier to good care. ${ }^{[1]}$ Among all severe forms of fears, which were ranked the fear for dental procedures was ranked the tenth. ${ }^{[2]}$ Trypanophobia (an irrational fear of procedures involving injections) was not uncommon among dental patients. ${ }^{[3]}$ Local anesthesia (LA) forms the backbone of pain control techniques in dentistry. The injection of cocaine with epinephrine in 1885 by William Halsted enabled, for the first time, surgical procedures to be performed painlessly in a conscious human being. ${ }^{[3]}$ Painless LA delivery is mandatory, especially when used for preschool children. ${ }^{[4]}$ To the best of knowledge, less studies on the influence of needle gauge in perception of pain during local dental anesthetic injection had been conducted on children. Therefore, the purpose of this study was to evaluate the children's pain perception and to record their reactions, while providing dental LA with 26 and 30 gauge needles.

\section{MATERIALS AND METHODS}

The present in vivo study was undertaken in the Department of Pedodontics and Preventive Dentistry. In Rajah Muthiah Dental College and Hospital, University, to compare in vivo the pain perception of children to LA using 26 gauge and 30 gauge needles. Thirty children ( 16 boys and 14 girls) who met the inclusion criteria were selected for participation in this study. Preoperative behavioral assessment using the Frankl et al. scale was done. ${ }^{[6]}$ Children were assigned into two groups:

Group A: 12 children aged 6-8 years (primary school age) Group B: 18 children aged 9-12 years (middle school age). 


\section{Inclusion criteria}

Children with treatment needs in two different quadrants either in maxillary or mandibular arch, children with multiple retained deciduous teeth, nonsymptomatic teeth requiring extraction or pulp therapy and asymptomatic teeth/root stumps requiring extraction were included. Healthy children with no prior dental treatment, requiring a minimum of two clinical appointments of similar operative procedures on both sides of the same jaw preceded by local anesthetic injection, none of which was due to emergency were included. Children who demonstrated positive or definitely positive behavior during pretreatment evaluation (ranking 3 or 4 in the Frankl scale), and none of them who needed premedication for receiving dental treatment were included in the present study.

\section{Exclusion criteria}

Children with any emergency treatment needs such as abscess, cellulitis and space infections, with past painful experience, children/parents not willing to participate and who were categorized under Frankl's negative or definitely negative in pre procedure behavior assessment were excluded.

All parents were informed about the treatments and treatment procedures, and informed consents were received before the procedures. The field of insertion was dried with cotton rolls. Lidocaine topical aerosol $15 \% \mathrm{w} / \mathrm{w}$ was actuated for two sprays and after $2 \mathrm{~min}$ LA was administered. Euphemisms such as "putting the tooth to sleep," were used to describe the injection to all the children. Distraction and conventional nonpharmacological techniques of behavior management were used. A random crossover design was used. Every child acted as one's own control, while receiving each treatment on the opposite side of the same arch. Each patient was randomly assigned to receive the injection either with a 26 or 30 gauge needle for the first visit, while the injection with the other needle was administered during the second visit. ${ }^{[5]}$ Rating scales were used for objective and subjective evaluation.

\section{Objective evaluation}

During the local anesthetic injection (2\% lidocaine - I in 100000 epinephrine) Taddio's et al. modified Behavioral Pain scale (1995) ${ }^{[7]}$ was used for objective pain signs and reactions of the children. The scale consisted of the following as parameters of pain. They are crying, facial display (eyes' squeezing), arm movement, leg movement and torso movements. They were recorded as either present or absent. Only two of the four of Craig's most descriptive facial actions such as eye brow bulge and eye squeeze were recorded, ${ }^{[8]}$ because during injection, the mouth was open and the nose was usually partially encased by the operator's hand. Taddio's scale scores were recorded and then later were analyzed. One assistant who was blinded to the type of injection and needle used, recorded the complete procedures in video recorder. All the injections were administered by the same operator who was not related the study. The injection techniques were determined by random selection. To ensure a constant rate of injection, the operator was trained to deliver at rate of $\mathrm{I} \mathrm{ml} / 2 \mathrm{~min}$. The assistant used the chronometer and recorded the duration of the infusion [Figs. I-3].

\section{Subjective evaluation}

Immediately, after the injections, children were asked to fill in the Wong-Baker FACES pain rating scale (FPS) for subjective evaluation of pain perception after the injection [Fig. 4]. The scale comprised of a row of six faces depicting the different pain experience by facial reactions. They were as follows: Face 0: Does not hurt at all. Face 2: Hurts just a little bit. Face 4: Hurts a little more. Face 6: Hurts even more. Face 8: Hurts a whole lot. Face 10: Hurts worst, although the child does not have to be crying to have this worst pain. The child was asked to select the face that best described how much pain he or she had. The scores above four were considered to be painful. ${ }^{9]}$ Verbal instructions were given to the child on how to utilize the FPS. The FPS measured the unpleasantness and dimension of a child's pain experience. ${ }^{[9]}$ the values for this scale ranged between 0 and 5 , where 0 was "no hurt" and 5 was "hurt very much." The objective and subjective behavioral parameters were recorded by Taddio's pain rating scale and FPS respectively and analyzed statistically by student $t$-test and Chi-squared analyses, and significance was set at $P<0.05$.

\section{RESULTS}

The mean pain score for 26 gauge needles was found to be 2.80 whereas for pain scores for 30 gauge needles was found to be 2.37. The mean difference was found to be statistically significant $(P=0.00 \mathrm{I})$ [Table I]. In the present study, most of the patients reported both LA using the 26 and 30 gauge needles to be nonpainful experience, that is, $70 \%$ (21 out of 30 ) and $93.3 \%$ (28 out of 30 ) respectively, which was evident from using the Wong-Baker's FPS [Table 2]. In the individual parameters and its percentage in 26 gauge and 30 gauge groups, most of the parameters were not expressed in 30 gauge group comparatively.

Table 1: Mean and SD for Taddio's pain scores in relation to 26 gauge group and $\mathbf{3 0}$ gauge group

\begin{tabular}{lccccc}
\hline Groups & $n$ & Mean & SD & $t$ value & $P$ value \\
\hline 26 gauge & 30 & 2.80 & 0.76 & 3.067 & 0.001 \\
30 gauge & 30 & 2.37 & 0.72 & & \\
\hline
\end{tabular}

SD: Standard deviation 
The mean Taddio's pain score for age group 6-8 years was found to be 3.00 and the mean pain score for age group $9-12$ years was found to be 2.67 in patients who received LA with 26 gauge needles. However mean difference was not statistically significant with a $P=0.29$. The mean Taddio's pain score for age group 6-8 years was found to be 2.39 and the mean pain score for age group $9-12$ years was found to be 2.33 in patients receiving LA with 30 gauge needles. However, the mean difference was not statistically significant with a $P=0.85$.

The mean pain score in males using 26 gauge needles was found to be 2.57 , whereas mean pain score in females using 26 gauge needles was found to be 3.00 , which was slightly higher, but was not statistically significant $(P=0.12)$. The mean pain score in males using 30 gauge needles was found to be 2.2I, whereas mean pain score in females using 30 gauge needles was found to be 2.50, which was slightly higher, but not statistically significant $(P=0.28)$.

The mean pain score for nerve block anesthesia for patients who received LA with 26 gauge needles was found to be $2.7 \mathrm{I}$, whereas the mean pain score for nerve blocks for patients who received LA with 30 gauge needles was found to be 2.21 There was a slight mean difference, but statistically not significant $(P=0.104)$ [Table 3]. The mean pain score for infiltration anesthesia with 26 gauge needles was found to be 2.93 whereas the mean pain score for infiltration anesthesia with 30 gauge needles was found to be 2.43 and statistically significant $(P=0.05)$ [Table 4].

The mean pain score in first visit for patients who received LA with 30 gauge needles was found to be 2.00 , whereas for second visit was found to be 2.61. The mean difference was found to be statistically significant $(P=0.0 \mathrm{I})$ [Table 5]. The mean pain score in first visit for patients who received LA with 26 gauge needles was found to be 2.89 , whereas for second visit was found to be 2.50 . The mean pain difference was however found to be statistically not significant $(P=0.127)$ [Table 6].

\section{DISCUSSION}

The mean pain scores for 26 gauge needles was found to be 2.80 whereas for pain scores for 30 gauge needles were found to be 2.37. The mean difference was found to be statistically significant $(P=0.00 \mathrm{I})$. This might be due to fact that the 30 gauge needle required significantly less force $(69 \mathrm{mN})$ than the 27 gauge needle $(139 \mathrm{mN}) \cdot{ }^{[10]}$ In analyzing the individual parameters $13.4 \%$ (4 out of 30 injections) of children cried in receiving anesthesia with 26 gauge needles and $3.4 \%$ ( 1 out of 30 injections) in receiving anesthesia with 30 gauge needles, which was in accordance with
Table 2: Pain experience using Wong-Baker's scale in percentage in relation to different gauge groups

\begin{tabular}{lcc}
\hline Groups & Painful experience (\%) & Nonpainful experience $(\%)$ \\
\hline 26 gauge & $9(30)$ & $21(70)$ \\
30 gauge & $2(6.7)$ & $28(93.3)$ \\
\hline
\end{tabular}

Table 3: Mean and SD of Taddio's pain scores for nerve blocks and their relation to gauge groups

\begin{tabular}{lccccc}
\hline Groups & $n$ & Mean & SD & $t$ value & $P$ value \\
\hline 26 gauge & 14 & 2.71 & 0.69 & 1.69 & 0.104 \\
30 gauge & 16 & 2.21 & 0.89 & & \\
\hline
\end{tabular}

SD: Standard deviation

Table 4: Mean and SD of Taddio's pain scores for infiltration and their relation to gauge groups

\begin{tabular}{lccccc}
\hline Groups & $n$ & Mean & SD & $t$ value & $P$ value \\
\hline 26 gauge & 16 & 2.93 & 0.80 & 2.03 & 0.05 \\
30 gauge & 14 & 2.43 & 0.51 & & \\
\hline
\end{tabular}

SD: Standard deviation

Table 5: Mean and SD of Taddio's pain score in first and second visits in $\mathbf{3 0}$ gauge

\begin{tabular}{lccccc}
\hline Groups & $n$ & Mean & SD & $t$ value & $P$ value \\
\hline First visit & 12 & 2.00 & 0.60 & 2.55 & 0.01 \\
Second visit & 18 & 2.61 & 0.70 & & \\
\hline
\end{tabular}

SD: Standard deviation

Table 6: Mean and SD of Taddio's pain score in first and second visits in 26 gauge

\begin{tabular}{lccccc}
\hline Groups & $n$ & Mean & SD & $t$ value & $P$ value \\
\hline First visit & 18 & 2.89 & 0.83 & 1.57 & 0.127 \\
Second visit & 12 & 2.50 & 0.52 & & \\
\hline
\end{tabular}

SD: Standard deviation

previous studies. ${ }^{[5]}$ In the present study most of the patients reported both LA using the 26 and 30 gauge needles to be nonpainful experience, that is, $70 \%$ (2I out of 30 ) and 93.3\% (28 out of 30 ) respectively using the Wong-Baker's FPS. This might be explained by the fact that in a good dentist - child rapport the child might want to satisfy the caregiver. In addition, the anesthetic was delivered very slowly, children did not see the needle and conventional non pharmacological behavioral management techniques like positive reinforcement and distraction were used. ${ }^{[5]}$ It is also important that a slower injection also be used to increase patient comfort during the administration of local anesthetic agents ${ }^{[6]}$. Other advantages of large-gauge needles include less chance of needle breakage or deflection and less pressure needed for aspiration. ${ }^{[12]}$

The mean pain difference was not statistically significant in different age groups selected. This result was in 


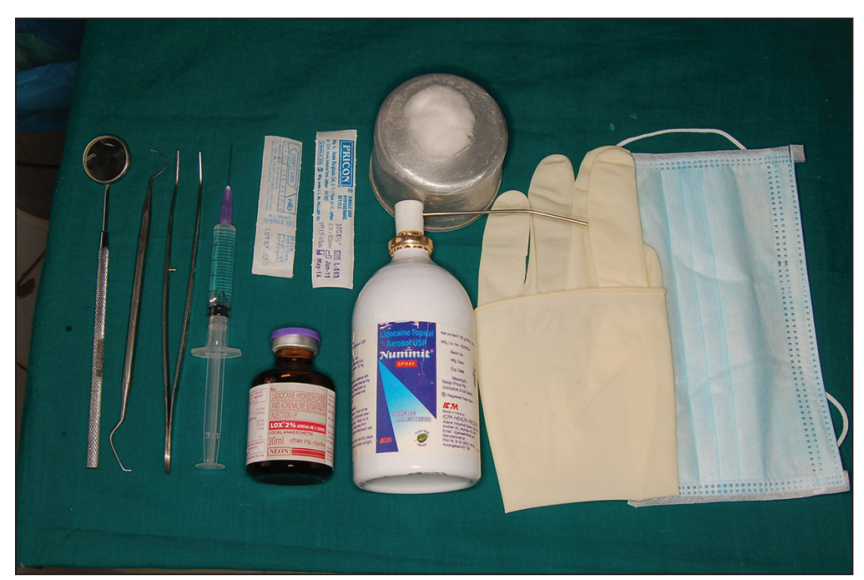

Figure 1: Armamentarium used

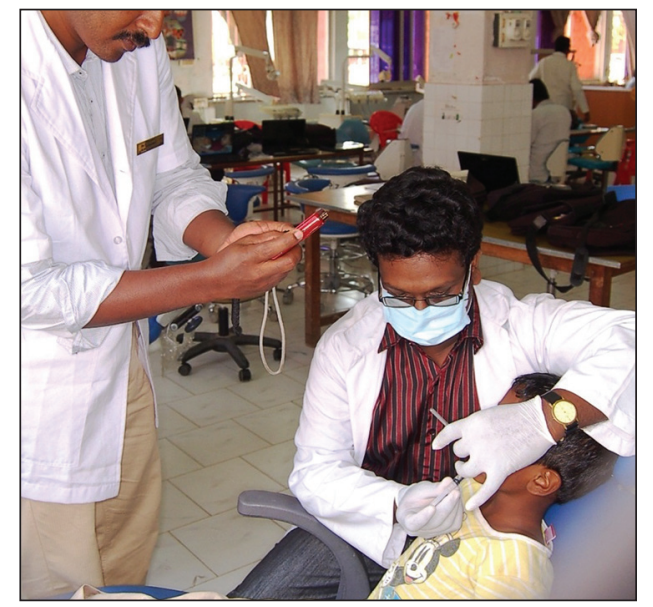

Figure 3: Recording objective pain signs and symptoms

accordance with previous studies. ${ }^{[5]}$ The slight difference between the age groups might be attributed to the following reasons: Older participants were more likely to use more problem solving and externalizing coping strategies than younger participants. ${ }^{[13]}$ also with increase in age, tolerance to cutaneous pain increased and tolerance to deep pain decreased. ${ }^{[14]}$ A young child responds more adversely to dental care. Anxious, uncooperative responses were most common in the youngest children and diminished in frequency as the child's age at the first visit increased. ${ }^{[15]}$

The mean pain scores were slightly higher in females than males but not statistically significant. The slight difference might be attributed to children with tendencies of seeking emotional support were more likely to exhibit lower pain tolerance, possibly due to habitual behavior of more intense pain expression. Females were more likely to use seeking emotional support as a coping strategy than males. ${ }^{\left[{ }^{[3]}\right.}$ It could also indicate that women knew, correctly, that they have a lower endurance level for pain. ${ }^{[7]}$ This might also be supported by the fact women were more likely than men to have been frightened while undergoing dental treatment ${ }^{[1]}$

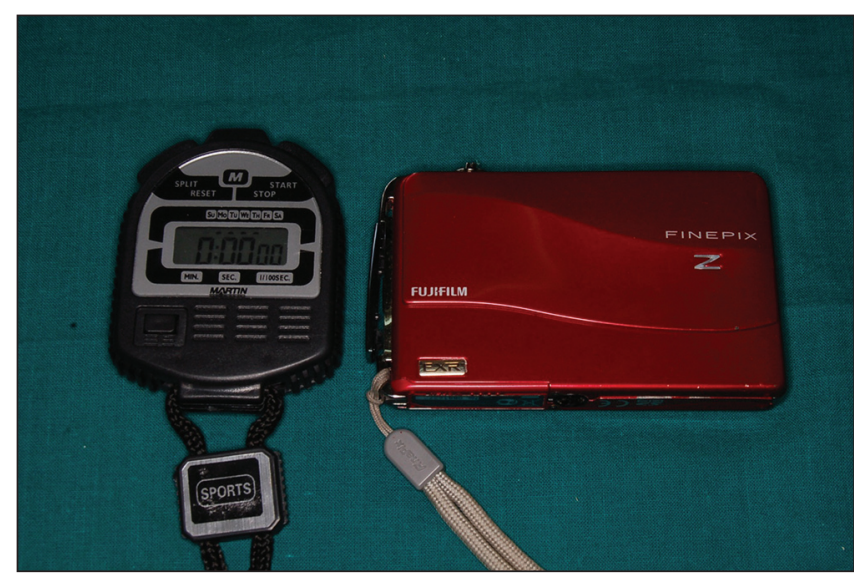

Figure 2: Recording and timing devices used

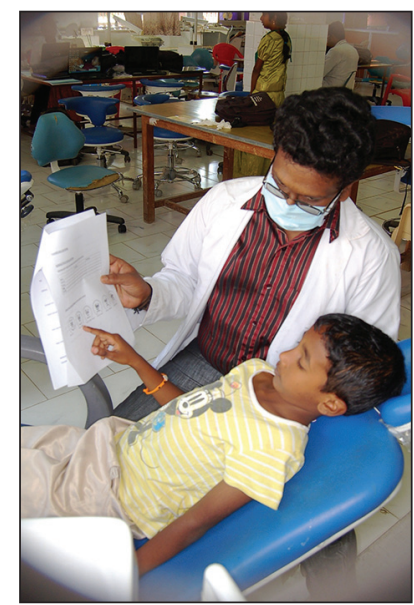

Figure 4: Registering subjective pain perception

The mean pain score for infiltration for patients who received LA with 26 gauge needles was found to be 2.93 , whereas the mean pain score for infiltration for patients who received LA with 30 gauge needles was found to be 2.43. The mean difference was found to be statistically significant $(P=0.05)$. The mean pain score for nerve block for patients who received LA with 26 gauge needles was found to be 2.7I, whereas the mean pain score for nerve blocks for patients who received LA with 30 gauge needles is found to be 2.21 There was a slight mean difference but statistically not significant $(P=0.104)$. This might be due to the fact that children do not think that inferior dental nerve block injections hurt very much ${ }^{[2]}$ and this also might be due to the different tissues through which the needle passes during the different techniques ${ }^{[5]}$ and also the area being injected in the oral cavity may have direct relation to pain and discomfort perception ${ }^{[1]}$. Oral mucosa and periodontal ligament have an abundant number of free nerve endings, whereas the sub mucosa area had fewer. ${ }^{[18]}$

The mean pain score in first visit for patients who received LA with 26 gauge needles was found to be 2.89 , whereas for second visit was found to be 2.50 . The mean pain 
difference was found to be statistically not significant $(P$ $=0.127)$. The mean pain score in first visit for patients who received LA with 30 gauge needles was found to be 2.00 , whereas for second visit was found to be 2.61 . The mean pain difference was found to be statistically significant $(P=0.0 \mathrm{I})$. The changing pattern of pain response across dental visits implied that the effects of dental experience were complex and that a period of growing sensitization precedes the child's eventual adaptation to stressful treatment procedures. ${ }^{[15]}$ The accuracy of patient's reports of pain experienced in the past might be suspected, and that dental anxiety might be slow to extinguish because the discrepancy between expected and experienced pain felt at one appointment may not be recalled accurately by anxious patients at their next appointment. ${ }^{[19]}$

The difference in first and second visits might also be probably reasoned out from the notion that the young patient brings to the initial dental experience internal characteristics, which might either facilitate or impede his/ her adaptation to the stress. Certain developmental and personality characteristics might predispose the young dental patient to perceptually distort the degree of threat present, and to unrealistically interpret the amount of discomfort actually experienced. ${ }^{[20]}$ The children who received 26 gauge needles (pain score average 2.89 ) at the first visit showed more pain scores even the second visit of the 30 gauge needles (pain score average 2.6I). Whereas children who received 30 gauge needles at first visit (pain score average 2.00 ) showed less pain scores for even 26 gauge needles (pain score average 2.50). These values bring about the relevance of psychological and initial pain experience component of the pain. Many factors such as anxiety, fear, trust, perceived control over painful stimulus, interpretation of the painful stimulation, and personality influence pain. ${ }^{[21]}$ Therefore, pain scores in the different gauge groups could not be solely attributed to the induced stimulus alone. However the major factor in any injection is the human element. It bears repeating that the patient and operator should be psychologically prepared, the equipment sterile, the anesthetic fresh, aspiration performed, and the deposition gentle, slow, and careful.. ${ }^{[21]}$

\section{CONCLUSION}

The present study concludes that pain perception was less and perceived dental LA was less unpleasant when administered with a 30 gauge needle than with a 26 gauge needle. The pain due to injection penetration may be controlled by using thinner gauge needles, but LA deposition rate should be as slow as possible to avoid pain. During a child's first dental visit, use of less pain provoking armamentarium like the thinner gauge needles would aid in relieving anxiety and desensitize the patient slowly. However the thinner gauge needles need to be tested for other properties such as deflection, tensile stress for usage along with slow LA systems such as Computer Controlled local Anesthetic Delivery System.

\section{REFERENCES}

1. Milgrom P, Coldwell SE, Getz T, Weinstein P, Ramsay DS. Four dimensions of fear of dental injections. J Am Dent Assoc 1997;128:756-66.

2. Aldosari AM. Dental fear among visitors of primary health care centres in Saudi Arabia. Odontostomatol Trop 1999;13:97-9.

3. Malamed SF, Reed K, Poorsattar S. Needle breakage: Incidence and prevention. Dent Clin North Am 2010;54:745-56.

4. Tahmassebi JF, Nikolaou M, Duggal MS. A comparison of pain and anxiety associated with the administration of maxillary local analgesia with Wand and conventional technique. Eur Arch Paediatr Dent 2009;10:77-82.

5. Ram D, Hermida B L, Amir E. Reaction of children to dental injection with 27-or 30-gauge needles. Int J Paediatr Dent 2007;17:383-7.

6. Frankl SN, Shiere FR, Fogels HR. Should the parent remain with the child in the dental operatory. J Dent Child 1962;29:150-63.

7. Taddio A, Nulman I, Koren BS, Stevens B, Koren G. A revised measure of acute pain in infants. J Pain Symptom Manage 1995;10:456-63.

8. Craig KD. The Facial Display of Pain: Measurement of Pain in Infants and Children. Seattle, WA: IASP Press; 1998. p. 103-21.

9. Wong DL, Baker CM. Pain in children: Comparison of assessment scales. Pediatr Nurs 1988;14:9-17.

10. Lehtinen R. Penetration of 27-and 30-gauge dental needles. Int J Oral Surg 1983;12:444-5.

11. Meechan JG, Cole B, Welbury RR. The influence of two different dental local anaesthetic solutions on the haemodynamic responses of children undergoing restorative dentistry: A randomised, single-blind, split-mouth study. Br Dent J 2001;190:502-4.

12. Flanagan T, Wahl MJ, Schmitt MM, Wahl JA. Size doesn't matter: Needle gauge and injection pain. Gen Dent 2007;55:216-7.

13. Lu Q, Tsao JC, Myers CD, Kim SC, Zeltzer LK. Coping predictors of children's laboratory-induced pain tolerance, intensity, and unpleasantness. J Pain 2007;8:708-17.

14. Woodrow KM, Friedman GD, Siegelaub AB, Collen MF. Pain tolerance: Differences according to age, sex and race. Psychosom Med 1972;34:548-56.

15. Venham LL, Goldstein M, Gaulin-Kremer E, Peteros K, Cohan J, Fairbanks J. Effectiveness of a distraction technique in managing young dental patients. Pediatr Dent 1981;3:7-11

16. Rollman GB, Abdel-Shaheed J, Gillespie JM, Jones KS. Does past pain influence current pain: Biological and psychosocial models of sex differences. Eur J Pain 2004;8:427-33.

17. Locker D, Shapiro D, Liddell A. Negative dental experiences and their relationship to dental anxiety. Community Dent Health 1996;13:86-92.

18. Kaufman E, Epstein JB, Naveh E, Gorsky M, Gross A, Cohen G. A survey of pain, pressure, and discomfort induced by commonly used oral local anesthesia injections. Anesth Prog 2005;52:122-7.

19. Kent G. Memory of dental pain. Pain 1985;21:187-94.

20. Venham LL, Murray P, Gaulin-Kremer E. Personality factors affecting the preschool child's response to dental stress. J Dent Res 1979;58:2, 46-51.

21. Nusstein J, Lee S, Reader A, Beck M, Weaver J. Injection pain and postinjection pain of the anterior middle superior alveolar injection administered with the Wand or conventional syringe. Oral Surg Oral Med Oral Pathol Oral Radiol Endod 2004;98:124-31.

22. Brownbill JW, Walker PO, Bourcy BD, Keenan KM. Comparison of inferior dental nerve block injections in child patients using 30-gauge and 25-gauge short needles. Anesth Prog 1987;34:215-9.

How to cite this article: Asokan A, Rao AP, Mohan G, Reddy NV, Kumar K. A pain perception comparison of intraoral dental anesthesia with 26 and 30 gauge needles in 6-12-year-old children. J Pediatr Dent 2014;2:56-60.

Source of Support: Nil. Conflict of Interest: None declared. 